2010 and 10/12/2016. The records were then histology matched.

The data was then analysed using the Anacondas ${ }^{\mathrm{TM}} 3$ distribution of Python, using numpy, pandas, matplotlib and seaborn to clean and prepare, plot and perform statistical analysis on the data.

Results 23837 colonoscopies were performed on 18489 individual adults during the study period.

544 procedures had to be excluded as they lacked an NHS number and couldn't be histology matched. 23293 procedures remained.

$50.4 \%$ of the procedures were performed on females. The median age was 64. Across all the procedures, 25.46\% were reported as entirely normal by the endoscopist. $3.04 \%$ of procedures contained a histologically confirmed cancer.

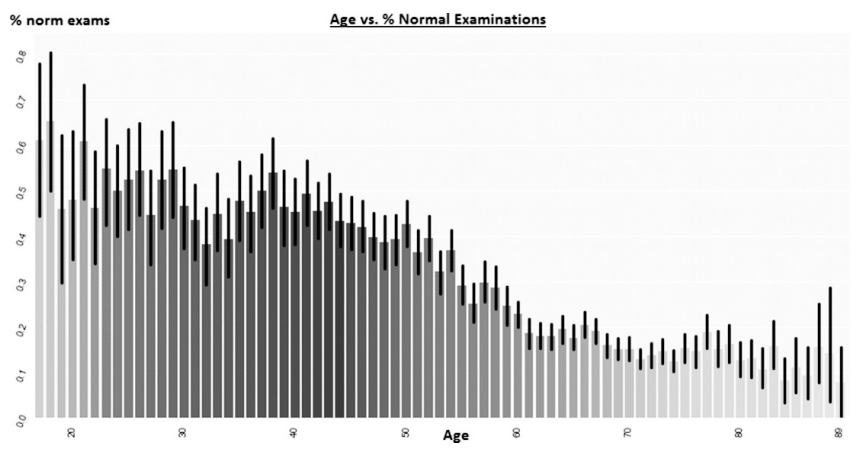

Abstract PTH-058 Figure 1 Age vs\% normal examinations

Age We found that the chances of obtaining a normal examination declined from $\sim 49 \% \pm 5 \% \leq 43$ years to $\leq 20 \% \pm 2 \%$ in those $\geq 61$ years.

In patients aged $\leq 43$ OR of a normal exam=3.29. For those aged $\geq 61$, (OR $=0.32$ for a normal exam). Note, all OR's in this study had $\mathrm{p}<0.0001$ significance.

Sex Examinations performed on females were more likely to be reported as normal compared to men $(\mathrm{OR}=1.73)$. For women $\leq 43$, OR for normality $=3.88$.

Priority Routine priority was strongly associated with normal colonoscopy, $(\mathrm{OR}=1.99)$. Routine procedures on females $\leq 43$ were very likely to be normal $(\mathrm{OR}=4.90)$. These patients were very unlikely to have cancer $(\mathrm{OR}=\mathbf{0 . 0 9 3})$.

Indications Abdominal pain, anaemia (iron deficient) and bowel habit changes (of all types) and family history of colonic cancer were all found to be associated with $\mathrm{a} \geq 40 \%$ rate of normal examinations, $(\mathrm{OR}=3.57)$.

The highest incidence of normal examinations was found amongst women $\leq 43$ undergoing routine colonoscopy for abdominal pain $(\mathrm{OR}=7.85)$, followed by bowel habit changes $(\mathrm{OR}=6.49)$, and anaemia $(\mathrm{OR}=5.91)$.

Conversely, the highest rates of pathology were found in men $\geq 61$ undergoing bowel cancer screening, (OR for pathology $=4.98$; OR for malignancy $=2$ ).

Conclusions We have developed a method for performing mass data analysis to identify trends in endoscopy data.

Our data can help improve future utilisation of other colonic investigational modalities like colon capsule for low risk individuals. This can release colonoscopy capacity for the patients most at need.

\section{PTH-059 ANALYSIS OF 140000 PATIENTS WITH BILE DUCT STONES PRESENTING TO ENGLISH ACUTE TRUSTS, 2013-2016}

${ }^{1}$ Maxime Delvincourt ${ }^{*},{ }^{2}$ Jodi Carter, ${ }^{2}$ Keith Bodger, ${ }^{3}$ George Webster, ${ }^{1}$ Richard Sturgess. ${ }^{1}$ Aintree University Hospital NHS Trust, Liverpool, UK; ${ }^{2}$ Methods Analytics, London, UK; ${ }^{3}$ University College London Hospital Trust, London, UK

10.1136/gutjnl-2018-BSGAbstracts.80

Common ile uct (CBD) stones 10\%-20\% of patients with gallstone disease. ndoscopic retrograde cholangio-pancreatography (ERCP)

Methods All adult patients with diagnosis (ICD-10) codes for alculus of bile duct with cholangitisalculus of bile duct with cholecystitisalculusofbile duct without cholangitis were used to select results from HospitalEpisode Statistics (HES) data for the years 2013/14 - 2016/17. Episodes were excluded where there were episodes with one or moreof these codes for the previous 2 financial years to censor patients already on a CBD pathway.

Results There were 140890 patients identified, of which $91178(64.72 \%)$ were female Mean age at first admission was 70. Of the 140890 individual patients, $102072(72 \%)$ had an emergency first admission. The incidence of cases in each year was 33,557, 35,053, 35621 and 36659 respectively. were performed on 48306 patients in this cohort over the study period of the 48306 individuals who had an ERCP, 39192 (65\%) had only one procedure, 7105 (1\%) had two procedures and $2009(\%)$ had three or more.

For the identified CBD cohort 60793 holecystectomies were performed over the study period with 17063 patients having had both an ERCP and holecystectomy.

\section{PTH-060 AN ASSESSMENT OF QUALITY OF, AND FACTORS AFFECTING, OESOPHAGOGASTRODUODENOSCOPY (OGD)}

David Tai ${ }^{\star}$, Gloria SZ Tun, Mustafa Jalal, Stefania Chetcuti Zammit, Mark McAlindon. Sheffield Teaching Hospitals NHS Trust, Sheffield, UK

\subsection{6/gutjnl-2018-BSGAbstracts.81}

Introduction Oesophagogastroduodenoscopy (OGD) is the accepted gold standard investigation for upper GI symptoms although quality has rarely been assessed. In this study, quality of OGD and the effects of sedation, procedural tolerance and duration of examination were investigated.

Methods Consecutive out-patients undergoing OGD were recruited. Patients scored (none-severe: 0-10) procedural distress, abdominal discomfort (AD) and pain (AP). Video recordings of the OGDs were reviewed, scoring the views (score 1$5:<25 \%, 25 \%-50 \%, 50 \%-75 \%,>75 \%$ and $100 \%$ of mucosa seen) of eight upper GI stations and time spent in retroflexion and in the duodenum. Scores and times are reported as mean ( \pm SEM).

Results OGD was performed on forty patients $(58 \pm 3$ years old, $62.5 \%$ male, $25 \%$ sedated) by one of three endoscopists. There was a significant difference between visualisation scores (oesophagus, $4.5 \quad( \pm 0.1)$; gastro-oesophageal junction, 4.5 $( \pm 0.1)$; cardia, $4.0( \pm 0.2)$; fundus, $3.7( \pm 0.2)$; upper body, $3.8( \pm 0.1)$; distal body, $4.1( \pm 0.1)$; incisura, $4.0( \pm 0.2)$ and antrum, $4.9( \pm 0.1)$; Kruskal-Wallis $\mathrm{H} \quad$ Test: $\mathrm{H}(7)=60.2$; 
$\mathrm{p}<0.001)$. Post-hoc analysis shows that the antrum was better visualised compared to all other gastric stations $(\mathrm{p}<0.001)$. When composite scores of fundus, antrum and upper body are compared to the distal body, incisura and antrum, views in the distal half of the stomach are significantly better visualised $\left(\chi^{2}=520, p=0.006\right)$. Procedural distress, AD and AP were $4.9( \pm 0.5), 2.8( \pm 0.5)$ and $1.3( \pm 0.3)$ respectively: none correlated with views in any station apart from those with more procedural $\mathrm{AD}$ who had poorer views of the distal gastric body $(\mathrm{r}=-0.36 \mathrm{p}=0.017)$. Sedation had no effect on distress, discomfort or pain or views of any station. Procedure time was $5 \mathrm{~m} 41 \mathrm{~s}( \pm 18)$ with $55 \mathrm{~s}( \pm 5)$ spent in retroflexion and $58 \mathrm{~s}( \pm 9)$ in the duodenum, none of which correlated with any visualisation scores. Time spent in the duodenum correlated with increasing procedural AP $(r=0.415, p=0.009)$. Patients $(25 \%)$ who said they would decline repeat OGD reported significantly greater AP $(3.0 \pm 0.9$ vs $0.7 \pm 0.3)$ and procedural distress $(7.7 \pm 0.5$ vs $4.0 \pm 0.6)$, but their visualisation scores, procedural times or use of sedation did not differ from those who would have a repeat test.

Conclusion Visualisation at OGD is variable, with excellent antral but comparatively poorer proximal gastric and incisura views. OGD is the cause of significant distress to patients, rather than discomfort or pain, although duodenal examination may be painful in some. There was little correlation of quality of views with sedation, tolerance or duration of examination.

\section{PTH-061 MISSED OESOPHAGOGASTRIC CANCER CORRELATES WITH HIGHER LIST INTENSITY BUT NOT RATES OF SEDATION}

David FW Tai* ${ }^{*}$ Andrew Hopper, Mark McAlindon. Sheffield Teaching Hospitals NHS Trust, Sheffield, UK

\subsection{6/gutjnl-2018-BSGAbstracts.82}

Introduction Oesophagogastric (OG) cancers diagnosed within three years of an unremarkable oesophagogastroduodenoscopy (OGD) can be considered a failure to earlier diagnose the OG cancer, or post-OGD upper GI cancer (POUGIC). Retrospective studies suggest that they comprise up to $11 \%$ of OG cancer diagnosis [Menon, Endosc Int Open 2014] and auditing rates of POUGIC is a recent quality standard for endoscopy units [Beg, Gut 2017]. We examined whether patient sedation or procedural burden affects the rate of POUGIC.

Methods Cases of OG cancer diagnosed at OGD between Jan 2013 and Dec 2016 at Sheffield Teaching Hospitals were identified from our upper GI cancer database. OGD performed up to three years prior to diagnostic OGD were reviewed to identify cases of POUGIC. Rates of sedation and number of procedural points (one for OGD; two for colonoscopy; plus one for therapeutics) on lists were compared in three groups: a) the index procedures, b) the diagnostic procedures and c) age, sex and endoscopist matched patient control procedures in which focal (mucosal or vascular) lesions (FL) were identified. FL were approximated in size and location (oesophageal or gastric) as a surrogate for an early neoplastic lesion.
Results A total of 553 patients $(64.2 \%$ male, mean age $72 \pm 1$, $50.4 \%$ gastric) were diagnosed with OG cancer. Forty $(7.2 \%$, mean age $74 \pm 2,55 \%$ male, $55 \%$ gastric,) patients had 47 non-diagnostic procedures up to three years prior to diagnosis. Mean time from index to diagnostic OGD was $486 \pm 55$ days. In $42.5 \%$ OGD was performed within one year of diagnosis. There was no difference in the age, gender and rates of sedation (25 vs $28.5 \%$ ) between patients at index and diagnostic procedures. At index OGD the sedation rates were higher (44.7 vs $26.3 \% \mathrm{p}=0.049$ ) than at diagnostic OGD but there was a greater number of procedural points on the list $(7.9$ vs $9.3 \mathrm{p}=0.007)$. Control patients $(\mathrm{n}=38$, mean age $72 \pm 2$ $\mathrm{p}=0.64$ compared to POUGIC patients) with FL had OGD done a median of 33 days ( -357 to 728 days) from the index OGD. No suitable controls were identified in 2 patients. There was no difference in the sedation rates $(25.0$ vs $26.3 \% \mathrm{p}=0.89$ ) but there was a trend towards a higher number of procedural points $(9.3$ vs $8.7 \mathrm{p}=0.057)$ between the index OGD for POUGIC patients and their controls.

Conclusions The local POUGIC rate is $7.2 \%$. No differences in sedation rate between index, diagnostic or control procedures with representative FL suggest use of sedation may not help detection of early neoplastic lesions. However, endoscopy lists with OGDs which miss OG cancer seem to have a heavier burden than ones that diagnosed cancer and other FL suggesting that reduced list intensity may reduce the likelihood of missed pathology.

\section{PTH-062 CLINIC BASED OUTPATIENT TRANSNASAL ENDOSCOPY:IMPLEMENTATION AND EVALUATION OF AN INNOVATIVE ENDOSCOPY SERVICE}

${ }^{1}$ Nilanjana Tewari ${ }^{*},{ }^{1}$ Adolfo Parra-Blanco, ${ }^{1}$ Sarmad Sami, ${ }^{1}$ Shiv Budihal, ${ }^{1}$ Nina Lewis, 1James Catton, ${ }^{1,2}$ Krish Ragunath. ${ }^{1}$ Nottingham University Hospitals NHS Trust, Nottingham, UK; ${ }^{2}$ Nottingham Digestive Diseases BRU, Nottingham, UK

\subsection{6/gutjnl-2018-BSGAbstracts.83}

Introduction There is increasing evidence that Transnasal endoscopy (TNE), performed with an ultrathin HD scope, is well tolerated with minimal cardiorespiratory stress and better patient experience than standard endoscopy. We report preliminary results from a new outpatient TNE service developed in a university teaching hospital which is a tertiary referral centre for gastroenterology and upper gastrointestinal surgery.

Methods After local governance approvals, TNE was introduced and performed by 5 experienced endoscopists. All procedures were performed in an outpatient clinic adjacent to the endoscopy recovery area over a 6 month period. Patients were assessed as suitable for TNE based on local guidelines and if agreeable, underwent TNE using Pentax EPK-i7000 HD video endoscopy processor and EG16-K10 Transnasal endoscope (outer diameter $5.4 \mathrm{~mm}, 2.0 \mathrm{~mm}$ instrument channel) under topical anaesthetic plus anti-congestant applied to nostril. An antifoam/mucolytic drink was given $15 \mathrm{~min}$ prior to procedure. If the nose could not be intubated, the patient was offered the procedure using the narrow endoscope trans-orally. Preliminary data was collected in a pilot study in which patients were asked to complete a visual analogue score (VAS) and 